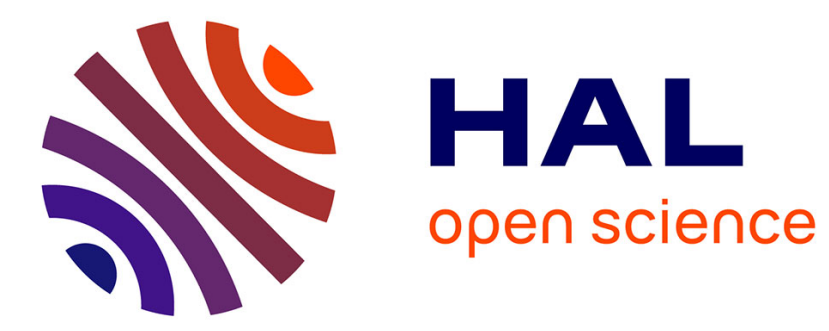

\title{
Magnetic moment manipulation by a Josephson current
}

François Konschelle, Alexandre I. Buzdin

\section{To cite this version:}

François Konschelle, Alexandre I. Buzdin. Magnetic moment manipulation by a Josephson current. Physical Review Letters, 2009, 102, pp.017001. 10.1103/PhysRevLett.102.017001 . hal-00333601v3

\section{HAL Id: hal-00333601 \\ https://hal.science/hal-00333601v3}

Submitted on 8 Jan 2009

HAL is a multi-disciplinary open access archive for the deposit and dissemination of scientific research documents, whether they are published or not. The documents may come from teaching and research institutions in France or abroad, or from public or private research centers.
L'archive ouverte pluridisciplinaire HAL, est destinée au dépôt et à la diffusion de documents scientifiques de niveau recherche, publiés ou non, émanant des établissements d'enseignement et de recherche français ou étrangers, des laboratoires publics ou privés.

\section{다(1) (2)}

Distributed under a Creative Commons Attribution - ShareAlikel 4.0 International 


\title{
Magnetic moment manipulation by a Josephson current
}

\author{
F. KONSChELle* and A. Buzdin \\ Condensed Matter Theory Group, CPMOH, Université de Bordeaux and CNRS. F-33405 Talence, France
}

(Dated: January 8, 2009, Document published in Phys. Rev. Lett. 102, 017001 (2009).)

\begin{abstract}
We consider a Josephson junction where the weak-link is formed by a non-centrosymmetric ferromagnet. In such a junction, the superconducting current acts as a direct driving force on the magnetic moment. We show that the a.c. Josephson effect generates a magnetic precession providing then a feedback to the current. Magnetic dynamics result in several anomalies of current-phase relations (second harmonic, dissipative current) which are strongly enhanced near the ferromagnetic resonance frequency.
\end{abstract}

Many interesting phenomena have been observed recently in the field of spintronics: the spin-dependent electric current and inversely the current-dependent magnetization orientation (see for example [1, 2]). Moreover, it is well known that spin-orbit interaction may be of primary importance for spintronic, namely for systems using a two-dimensional electron gas [3]. In the superconductor/ferromagnet/superconductor (S/F/S) Josephson junctions, the spin-orbit interaction in a ferromagnet without inversion symmetry provides a mechanism for a direct (linear) coupling between the magnetic moment and the superconducting current 4 . Similar anomalous properties have been predicted for Josephson junctions with spin-polarized quantum point contact in a two dimensional electron gas [5]. S/F/S junctions are known to reveal a transition to $\pi$-phase, where the superconducting phase difference $\varphi$ in the ground state is equal to $\pi$ [6]. However, the current-phase relation (CPR) in such a $\pi$-junction has a usual sinusoidal form $I=I_{c} \sin \varphi$, where the critical current $I_{c}$ depends in a damped oscillatory manner on the modulus of the ferromagnet exchange field. In a non-centrosymmetric ferromagnetic junction, called hereafter $\varphi_{0}$-junction, the time reversal symmetry is broken and the CPR becomes $I=I_{c} \sin \left(\varphi-\varphi_{0}\right)$, where the phase shift $\varphi_{0}$ is proportional to the magnetic moment perpendicular to the gradient of the asymmetric spin-orbit potential [4]. Therefore, manipulation of the internal magnetic moment can be achieved via the superconducting phase difference (i.e. by Josephson current).

In the present work we study theoretically the spin dynamics associated with such $\varphi_{0}$-junctions. Though there is a lot of experimental progress in studying the static properties of $\mathrm{S} / \mathrm{F} / \mathrm{S}$ junctions, little is known about the spin-dynamics in $\mathrm{S} / \mathrm{F}$ systems. Note here the pioneering work [7] where a sharpening of the ferromagnetic resonance was observed below the superconducting transition in $\mathrm{Nb} / \mathrm{Ni}_{80} \mathrm{Fe}_{20}$ system. Theoretically, the single spin dynamics interplay with a Josephson effect has been studied in 8, 9, 10, 11. More recently, the dynamically induced triplet proximity effect in $\mathrm{S} / \mathrm{F} / \mathrm{S}$ junctions was studied in [12, 13, while the junctions with composite regions (including several $\mathrm{F}$ regions with different magnetization) were discussed in [14, 15]. Here we consider a simple $\mathrm{S} / \mathrm{F} / \mathrm{S} \varphi_{0}$-junction in a low frequency regime $\hbar \omega_{J} \ll T_{c}$
$\left(\omega_{J}=2 e V / \hbar\right.$ being the Josephson angular frequency [16]), which allows us to use the quasi-static approach to treat the superconducting subsystem in contrast with the case analyzed in [12, 13. We demonstrate that a d.c. superconducting current may produce a strong orientation effect on the $\mathrm{F}$ layer magnetic moment. More interestingly, the a.c. Josephson effect, i.e. applying a d.c. voltage $V$ to the $\varphi_{0}$-junction, would produce current oscillations and consequently magnetic precession. This precession may be monitored by the appearance of higher harmonics in CPR as well as a d.c. component of the current. In particular regimes, a total reversal of the magnetization could be observed. In the case of strong coupling between magnetic and superconducting subsystems, complicated non-linear dynamic regimes emerge.

To demonstrate the unusual properties of the $\varphi_{0^{-}}$ junction, we consider the case of an easy-axis magnetic anisotropy of the F material (see Fig.11). Both the easy axis and gradient of the asymmetric spin-orbit potential $\mathbf{n}$ are along the $z$-axis. Note that suitable candidates for the $\mathrm{F}$ interlayer may be $\mathrm{MnSi}$ or FeGe. In these systems, the lack of inversion center comes from the crystalline structure, but the origin of broken-inversion symmetry may also be extrinsic, like in a situation near the surface of a thin F film. In the following, we completely disregard the magnetic induction. Indeed the magnetic induction in the $(x y)$ plane is negligible for the thin $\mathrm{F}$ layer considered in this paper, whereas the demagnetization factor cancels the internal induction along the $z$-axis $(N=1)$. The coupling between $\mathrm{F}$ and $\mathrm{S}$ subsystems due to the orbital effect has been studied in [17] and it appears to be very weak, and quadratic over magnetic moment $\mathbf{M}$ for the case when the flux of $\mathbf{M}$ through the $\mathrm{F}$ layer is small in comparison with flux quantum $\Phi_{0}=h / 2 e$.

The superconducting part of the energy of a $\varphi_{0^{-}}$ junction is

$$
E_{s}\left(\varphi, \varphi_{0}\right)=E_{J}\left[1-\cos \left(\varphi-\varphi_{0}\right)\right],
$$

where $E_{J}=\Phi_{0} I_{c} / 2 \pi$ is the Josephson energy, $I_{c}$ is the critical current and $\varphi_{0}$ is proportional to the $M_{y}$ component of the magnetic moment (see Fig.11). Therefore, when the magnetic moment is oriented along the $z$-axis, we have the usual Josephson junction with $\varphi_{0}=0$. Assuming the ballistic limit we may estimate the charac- 


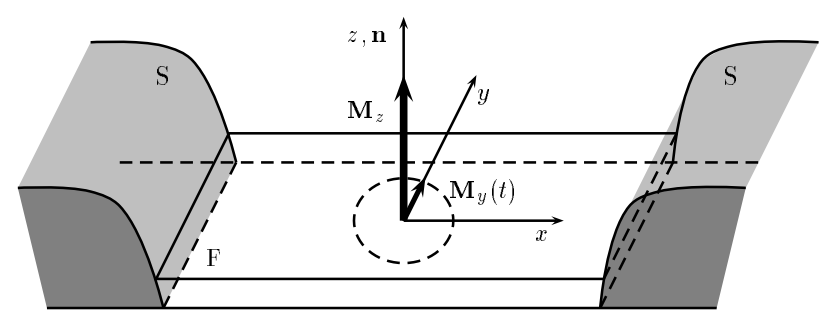

FIG. 1: Geometry of the considered $\varphi_{0}$-junction. The ferromagnetic easy-axis is directed along the $z$-axis, which is also the direction $\mathbf{n}$ of the gradient of the spin-orbit potential. The magnetization component $\mathbf{M}_{y}$ is coupled with Josephson current through the phase shift term $\varphi_{0} \propto \mathbf{n}$. $(\mathbf{M} \wedge \nabla \Psi)$, where $\Psi$ is the superconducting order parameter $(\nabla \Psi$ is along the $x$-axis in the system considered here).

teristic Josephson energy as [6] $\Phi_{0} I_{c} / S \sim T_{c} k_{F}^{2} \sin \ell / \ell$ with $\ell=4 h L / \hbar v_{F}$, where $S, L$ and $h$ are the section, the length and the exchange field of the $\mathrm{F}$ layer, respectively. The phase shift is

$$
\varphi_{0}=\ell \frac{v_{\mathrm{so}}}{v_{F}} \frac{M_{y}}{M_{0}}
$$

where the parameter $v_{\mathrm{so}} / v_{F}$ characterizes the relative strength of the spin-orbit interaction 四. Further on we assume that $v_{\mathrm{so}} / v_{F} \sim 0.1$. If the temperature is well below the Curie temperature, $M_{0}=\|\mathbf{M}\|$ can be considered as a constant equal to the saturation magnetization of the $\mathrm{F}$ layer. The magnetic energy contribution is reduced to the anisotropy energy

$$
E_{M}=-\frac{K \mathcal{V}}{2}\left(\frac{M_{z}}{M_{0}}\right)^{2}
$$

where $K$ is an anisotropy constant and $\mathcal{V}$ is the volume of the $\mathrm{F}$ layer.

Naturally, we may expect that the most interesting situation corresponds to the case when the magnetic anisotropy energy does not exceed too much the Josephson energy. From the measurements [18] on permalloy with very weak anisotropy, we may estimate $K \sim$ $4.10^{-5} \mathrm{~K} . \AA^{-3}$. On the other hand, typical value of $L$ in $\mathrm{S} / \mathrm{F} / \mathrm{S}$ junction is $L \sim 10 \mathrm{~nm}$ and $\sin \ell / \ell \sim 1$. Then, the ratio of the Josephson over magnetic energy would be $E_{J} / E_{M} \sim 100$ for $T_{c} \sim 10 \mathrm{~K}$. Naturally, in the more realistic case of stronger anisotropy this ratio would be smaller but it is plausible to expect a great variety of regimes from $E_{J} / E_{M} \ll 1$ to $E_{J} / E_{M} \gg 1$.

Let us now consider the case when a constant current $I<I_{c}$ is applied to the $\varphi_{0}$-junction. The total energy is (see, e.g. 16]):

$$
E_{\mathrm{tot}}=-\frac{\Phi_{0}}{2 \pi} \varphi I+E_{s}\left(\varphi, \varphi_{0}\right)+E_{M}\left(\varphi_{0}\right),
$$

and both the superconducting phase shift difference $\varphi$ and the rotation of the magnetic moment $M_{y}=M_{0} \sin \theta$ (where $\theta$ is the angle between the $z$-axis and the direction of $\mathbf{M}$ ) are determined from the energy minimum conditions $\partial_{\varphi} E_{\text {tot }}=\partial_{\varphi_{0}} E_{\text {tot }}=0$. It results in

$$
\sin \theta=\frac{I}{I_{c}} \Gamma \text { with } \Gamma=\frac{E_{J}}{K \mathcal{V}} \ell \frac{v_{\mathrm{so}}}{v_{F}},
$$

which signifies that a superconducting current provokes the rotation of the magnetic moment $M_{y}$ in the $(y z)$ plane. Therefore, for small values of the rotation, $\theta(I)$ dependence is linear. In principle, the parameter $\Gamma$ can be larger than one. In that case, when the condition $I / I_{c} \geq 1 / \Gamma$ is fulfilled, the magnetic moment will be oriented along the $y$-axis. Therefore, applying a d.c. superconducting current switches the direction of the magnetization, whereas applying an a.c. current on a $\varphi_{0}$-junction could generate the precession of the magnetic moment.

We briefly comment on the situation when the direction of the gradient of the spin-orbit potential is perpendicular (along $y$ ) to the easy axis $z$. To consider this case we simply need to take $\varphi_{0}=\ell\left(v_{\text {so }} / v_{F}\right) \cos \theta$. The total energy (4) has two minima $\theta=(0, \pi)$, while applying the current removes the degeneracy between them. However, the energy barrier exists for the switch from one minimum into another. This barrier may disappear if $\Gamma>1$ and the current is large enough $I>I_{c} / \Gamma$. In this regime the superconducting current would provoke the switching of the magnetization between one stable configuration $\theta=0$ and another $\theta=\pi$. This corresponds to the transitions of the junction between $+\varphi_{0}$ and $-\varphi_{0}$ states. The read-out of the state of the $\varphi_{0}$-junction may be easily performed if it is a part of some SQUID-like circuit (the $\varphi_{0}$-junction induces a shift of the diffraction pattern by $\left.\varphi_{0}\right)$.

In fact, the voltage-biased Josephson junction, and thus the a.c. Josephson effect provides an ideal tool to study magnetic dynamics in a $\varphi_{0}$-junction. In such a case, the superconducting phase varies with time like $\varphi(t)=\omega_{J} t$ 19. If $\hbar \omega_{J} \ll T_{c}$, one can use the static value for the energy of the junction (4) considering $\varphi(t)$ as an external potential. The magnetization dynamics are described by the Landau-Lifshitz-Gilbert equation (LLG) 20

$$
\frac{d \mathbf{M}}{d t}=\gamma \mathbf{M} \times \mathbf{H}_{\mathrm{eff}}+\frac{\alpha}{M_{0}}\left(\mathbf{M} \times \frac{d \mathbf{M}}{d t}\right),
$$

where $\mathbf{H}_{\text {eff }}=-\delta F / \mathcal{V} \delta \mathbf{M}$ is the effective magnetic field applied to the compound, $\gamma$ the gyromagnetic ratio, and $\alpha$ a phenomenological damping constant. The corresponding free energy $F=E_{s}+E_{M}$ yields

$$
\mathbf{H}_{\mathrm{eff}}=\frac{K}{M_{0}}\left[\Gamma \sin \left(\omega_{J} t-r \frac{M_{y}}{M_{0}}\right) \hat{\boldsymbol{y}}+\frac{M_{z}}{M_{0}} \hat{\boldsymbol{z}}\right],
$$

where $r=\ell v_{\mathrm{so}} / v_{F}$. Introducing $m_{i}=M_{i} / M_{0}, \tau=\omega_{F} t$ 

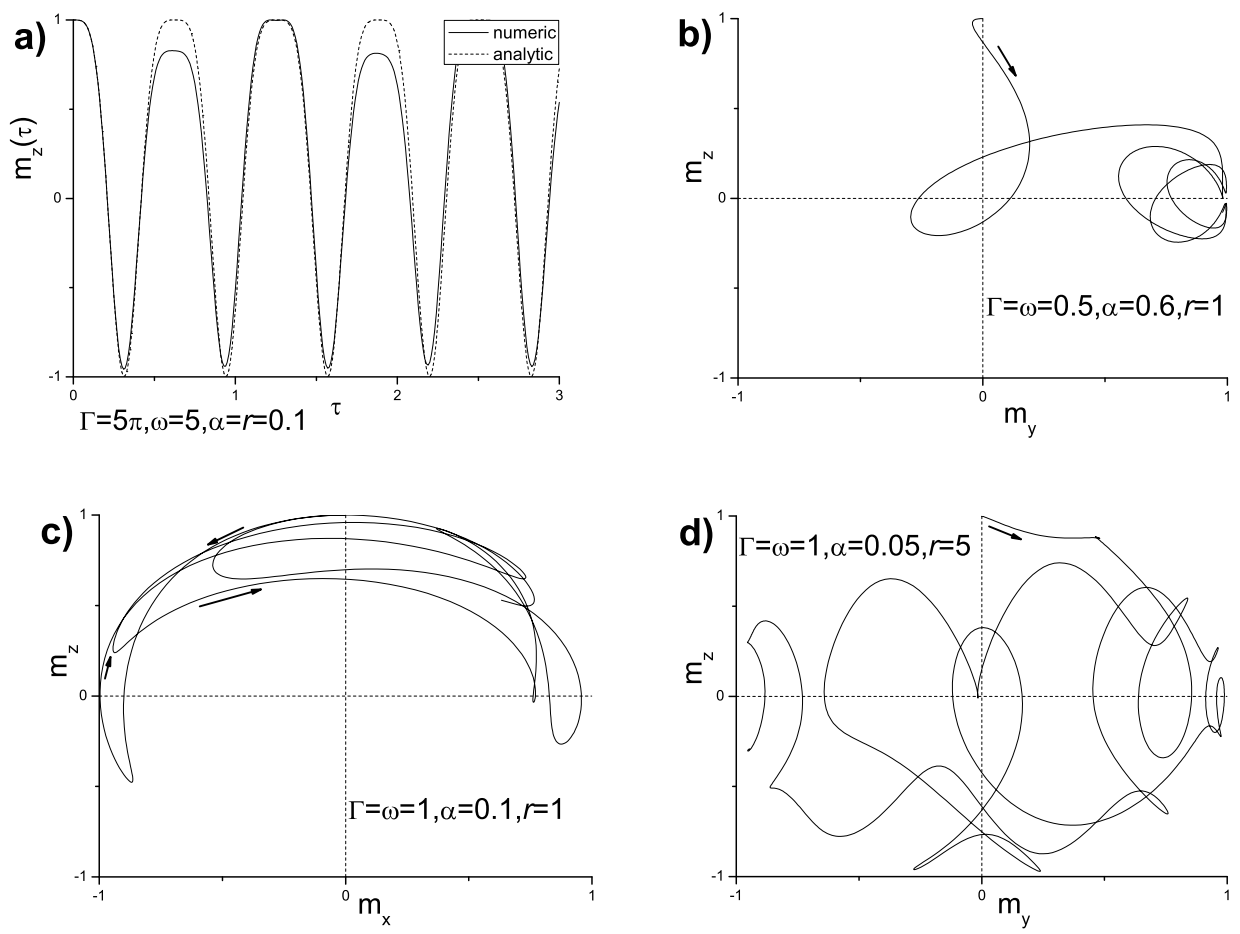

FIG. 2: Results of numerical analysis of the magnetic moment dynamics of the $\varphi_{0}$-junction. a) Reversal of $m_{z}$ from analytical expression Eq.(15) (dashed curve) and numerical one (normal curve). The other curves are related to the $\mathbf{M}$ trajectory: b) in strong damping case c) and d) in the strong coupling regime revealing incomplete and complete magnetic moment reversal, respectively.

$\left(\omega_{F}=\gamma K / M_{0}^{2}\right.$ is the frequency of the ferromagnetic resonance) in LLG equation (6) leads to

$$
\left\{\begin{array}{l}
\dot{m}_{x}=m_{z}(\tau) m_{y}(\tau)-\Gamma m_{z}(\tau) \sin \left(\omega \tau-r m_{y}\right) \\
\dot{m}_{y}=-m_{z}(\tau) m_{x}(\tau) \\
\dot{m}_{z}=\Gamma m_{x}(\tau) \sin \left(\omega \tau-r m_{y}\right)
\end{array}\right.
$$

where $\omega=\omega_{J} / \omega_{F}$. The generalization of Eq.(8) for $\alpha \neq 0$ is straightforward. One considers first the "weak coupling" regime $\Gamma \ll 1$ when the Josephson energy $E_{J}$ is small in comparison with the magnetic energy $E_{M}$. In this case, the magnetic moment precess around the $z$ axis. If the other components verify $\left(m_{x}, m_{y}\right) \ll 1$, then the equations (8) may be linearized, and the corresponding solutions are

$$
m_{x}(t)=\frac{\Gamma \omega \cos \omega_{J} t}{1-\omega^{2}} \text { and } m_{y}(t)=-\frac{\Gamma \sin \omega_{J} t}{1-\omega^{2}} .
$$

Near the resonance $\omega_{J} \approx \omega_{F}$, the conditions of linearization are violated and it is necessary to take the damping into account. The precessing magnetic moment influences the current through the $\varphi_{0}$-junction like

$$
\frac{I}{I_{c}}=\sin \omega_{J} t+\frac{\Gamma r}{2} \frac{1}{\omega^{2}-1} \sin 2 \omega_{J} t+\ldots,
$$

i.e., in addition to the first harmonic oscillations, the current reveals higher harmonics contributions. The amplitude of the harmonics increases near the resonance and changes its sign when $\omega_{J}=\omega_{F}$. Thus, monitoring the second harmonic oscillations of the current would reveal the dynamics of the magnetic system.

The damping plays an important role in the dynamics of the considered system. It results in a d.c. contribution to the Josephson current. Indeed, the corresponding expression for $m_{y}(t)$ in the presence of damping becomes

$$
m_{y}(t)=\frac{\omega_{+}-\omega_{-}}{r} \sin \omega_{J} t+\frac{\alpha_{-}-\alpha_{+}}{r} \cos \omega_{J} t
$$

where

$$
\omega_{ \pm}=\frac{\Gamma r}{2} \frac{\omega \pm 1}{\Omega_{ \pm}} \text {and } \alpha_{ \pm}=\frac{\Gamma r}{2} \frac{\alpha}{\Omega_{ \pm}}
$$

with $\Omega_{ \pm}=(\omega \pm 1)^{2}+\alpha^{2}$. It thus exhibits a damped resonance as the Josephson frequency is tuned to the ferromagnetic one $\omega \rightarrow 1$. Moreover, the damping leads to the appearance of out of phase oscillations of $m_{y}(t)$ (term proportional to $\cos \omega_{J} t$ in Eq.(11)). In the result the current

$$
\begin{aligned}
I(t) \approx I_{c} \sin \omega_{J} t & +I_{c} \frac{\omega_{+}-\omega_{-}}{2} \sin 2 \omega_{J} t+ \\
& +I_{c} \frac{\alpha_{-}-\alpha_{+}}{2} \cos 2 \omega_{J} t+I_{0}(\alpha)
\end{aligned}
$$


acquires a d.c. component

$$
I_{0}(\alpha)=\frac{\alpha \Gamma r}{4}\left(\frac{1}{\Omega_{-}}-\frac{1}{\Omega_{+}}\right) .
$$

This d.c. current in the presence of a constant voltage $V$ applied to the junction means a dissipative regime which can be easily detected. In some aspect, the peak of d.c. current near the resonance is reminiscent of the Shapiro steps effect in Josephson junctions under external r.f. fields. Note that the presence of the second harmonic in $I(t)$ Eq. 13) should also lead to half-integer Shapiro steps in $\varphi_{0}$-junctions [21].

The limit of the "strong coupling" $\Gamma \gg 1$ (but $r \ll 1$ ) can also be treated analytically. In this case, $m_{y} \approx 0$ and solutions of Eq.(8) yields

$$
\left\{\begin{array}{l}
m_{x}(t)=\sin \left[\frac{\Gamma}{\omega}\left(1-\cos \omega_{J} t\right)\right] \\
m_{z}(t)=\cos \left[\frac{\Gamma}{\omega}\left(1-\cos \omega_{J} t\right)\right]
\end{array},\right.
$$

which are the equations of the magnetization reversal, a complete reversal being induced by $\Gamma / \omega>\pi / 2$. Strictly speaking, these solutions are not exact oscillatory functions in the sense that $m_{z}(t)$ turns around the sphere center counterclockwise before reversing its rotation, and returns to the position $m_{z}(t=0)=1$ clockwise, like a pendulum in a spherical potential (see Fig.2.c).

Finally, we have performed numerical studies of the non-linear LLG Eq.(6) for some choices of the parameters when the analytical approaches fail. To check the consistency of our numerical and analytical approaches, we present in Fig.2.a) the corresponding $m_{z}(t)$ dependences for low-damping regimes. They clearly demonstrate the possibility of the magnetization reversal. In Figs.2 $2 \mathrm{~b}-\mathrm{d}$ ), some trajectories of the magnetization vectors are presented for general coupling regimes. These results demonstrate that the magnetic dynamics of $\mathrm{S} / \mathrm{F} / \mathrm{S}$ $\varphi_{0}$-junction may be pretty complicated and strongly nonharmonic.

If the $\varphi_{0}$-junction is exposed to a microwave radiation at angular frequency $\omega_{1}$, the physics that emerge are very rich. First, in addition to the Shapiro steps at $\omega_{J}=n \omega_{1}$, half-integer-steps will appear. Secondly, the microwave magnetic field may also generate an additional magnetic precession with $\omega_{1}$ frequency. Depending on the parameters of $\varphi_{0}$-junction and the amplitude of the microwave radiation the main precession mechanism may be related either to the Josephson current or the microwave radiation. In the last case the magnetic spin-orbit coupling may substantially contribute to the amplitude of the Shapiro steps. Therefore, we could expect a dramatic increase of this amplitude at frequencies near the ferromagnetic resonance. When the influence of the microwave radiation and Josephson current on the precession is comparable, a very complicated regime may be observed.

In the present work we considered the case of the easyaxis magnetic anisotropy. If the ferromagnet presents an easy-plane anisotropy than qualitatively the main conclusions of this article remain the same because the coupling between magnetism and superconductivity depends only on the $M_{y}$ component. However, the detailed dynamics would be strongly affected by a weak in-plane anisotropy.

To summarize, we have demonstrated that $\mathrm{S} / \mathrm{F} / \mathrm{S} \varphi_{0^{-}}$ junctions provide the possibility to generate magnetic moment precession via Josephson current. In the regime of strong coupling between magnetization and current, magnetic reversal may also occur. These effects have been studied analytically and numerically. We believe that the discussed properties of the $\varphi_{0}$-junctions could open interesting perspectives for its applications in spintronics.

The authors are grateful to Z. Nussinov, J. Cayssol, M. Houzet, D. Gusakova, M. Roche and D. Braithwaite for useful discussions and comments. This work was supported by the French ANR Grant N ${ }^{\circ}$ ANR-07-NANO011: ELEC-EPR.

* Electronic address: f.konschelle@cpmoh.u-bordeaux1.fr

$\uparrow$ Also at Institut Universitaire de France.

[1] I. Žutić, J. Fabian, and S. Das Sarma. Rev. Mod. Phys. 76, 323 (2004).

[2] J. Hauptmann, J. Paaske, and P. Lindelof. Nature Physics 4, 373 (2008).

[3] R. Winkler. Spin-orbit coupling effects in twodimensional electron and hole systems (Springer, New York, 2003).

[4] A. Buzdin. Phys. Rev. Lett. 101, 107005 (2008).

[5] A. A. Reynoso et al. Phys. Rev. Lett. 101, 107001 (2008).

[6] A. I. Buzdin. Rev. Mod. Phys. 77, 935 (2005).

[7] C. Bell et al. Phys. Rev. Lett. 100, 047002 (2008).

[8] J.-X. Zhu, and A. V. Balatsky. Phys. Rev. B 67, 174505 (2003).

[9] L. Bulaevskii et al. Phys. Rev. Lett. 92, 177001 (2004).

[10] J.-X. Zhu et al. Phys. Rev. Lett. 92, 107001 (2004).

[11] Z. Nussinov et al. Phys. Rev. B 71, 214520 (2005).

[12] S. Takahashi et al. Phys. Rev. Lett. 99, 057003 (2007).

[13] M. Houzet. Phys. Rev. Lett. 101, 057009 (2008).

[14] X. Waintal, and P. W. Brouwer. Phys. Rev. B 65, 054407 (2002).

[15] V. Braude, and Y. M. Blanter. Phys. Rev. Lett. 100, 207001 (2008).

[16] K. K. Likharev. Dynamics of Josephson junctions and circuits (Gordon and Beach Science Publishers, 1986).

[17] S. Hikino et al. J. Phys. Soc. Jap. 77, 053707 (2008).

[18] A. Y. Rusanov et al. Phys. Rev. Lett. 93, 057002 (2004).

[19] B. D. Josephson. Superconductivity (in two volumes), vol. 1, chapter 9, Weakly coupled superconductors (R.D Parks, Marcel Dekker, Inc., 1968). 
[20] E. M. Lifshitz, and L. P. Pitaevskii. Course of theoretical physics, Volume 9: Theory of the condensed state (Butterworth Heinemann, 1991).
[21] H. Sellier et al. Phys. Rev. Lett. 92, 257005 (2004). 